\title{
Broca's region: battles are not won by ignoring half of the facts
}

\author{
Roel M. Willems ${ }^{1}$ and Peter Hagoort ${ }^{1,2}$
}

${ }^{1}$ Donders Institute for Brain, Cognition and Behaviour, Centre for Cognitive Neuroimaging, Radboud University Nijmegen, P.O. Box 9101, 6500 HB Nijmegen, The Netherlands

${ }^{2}$ Max Planck Institute for Psycholinguistics, Wundtlaan 1, 6525 XD Nijmegen, The Netherlands

We welcome opinions about the role of Broca's area in higher-order cognition. Nevertheless, it was with somewhat of a surprise that we read the recent contribution by Grodzinsky and Santi [1] on this topic. Although they write that 'Broca's region might well be multifunctional' [1], Grodzinsky and Santi nevertheless conclude that the main role of Broca's region (more specifically Brodmann's areas 44 and 45) in language comprehension is related to 'syntactic movement'. Indefrey and colleagues [2] have shown that Broca's region has a role in syntactic encoding during language production, a result that could not be accounted for in terms of syntactic movement.

Nevertheless, even if one restricted discussion to language comprehension, it is quite remarkable that Grodzinsky and Santi [1] have ignored one set of highly relevant data points. Many neuroimaging studies show the involvement of Broca's region in semantic aspects of language understanding. For instance, Rodd and colleagues [3] showed that inferior frontal cortex (including BA 44 and 45 [4]) is sensitive to whether words in a sentence have multiple meanings or not. Sentences, such as 'There were dates and pears in the kitchen bowl' led to a stronger increase in activation in left inferior frontal cortex (including Broca's region) compared with sentences without homophones (e.g. 'There was beer and cider on the kitchen shelf'). In our own work we showed that an increase in the semantic integration load of spoken words or co-speech gestures leads to increased activation in Broca's region $[5,6]$. We want to stress that we are not referring to a finding from only a few studies. In a recent review we showed that 14 out of 16 neuroimaging studies on semantic aspects of sentence comprehension found reliable activation of left inferior frontal cortex (including, but not restricted to, BA44 and 45) [7] (see also Ref. [8]).

From a broader perspective, the fact that Grodzinsky and Santi [1] wobble between a multifunctional account of Broca's region and a very specific account, illustrates the core of the problem. It is questionable whether cortical regions like Broca's region follow the 'one-area-one-function rule'. Instead, Broca's region might be part of multiple networks, in which it is the network as a whole, rather than the single node, which determines its function [9] (see Ref. [10] for discussion).

In short, it seems that assigning a syntax-specific role to Broca's region in language understanding is premature and unwarranted given the available evidence. We propose to stop battling over which single (linguistic) function is to be assigned to Broca's region. Only if we consider all of the relevant data about the role of Broca's region, will we be able to arrive at a more adequate account of its role in language processing and other cognitive domains. No battle is ever won by ignoring half of the facts.

\section{References}

1 Grodzinsky, Y. and Santi, A. (2008) The battle for Broca's region. Trends Cogn. Sci. 12, 474-480

2 Indefrey, P. et al. (2001) A neural correlate of syntactic encoding during speech production. Proc. Natl. Acad. Sci. U. S. A. 98, 5933-5936

3 Rodd, J.M. et al. (2005) The neural mechanisms of speech comprehension: fMRI studies of semantic ambiguity. Cereb. Cortex $15,1261-1269$

4 Amunts, K. et al. (1999) Broca's region revisited: cytoarchitecture and intersubject variability. J. Comp. Neurol. 412, 319-341

5 Hagoort, P. et al. (2004) Integration of word meaning and world knowledge in language comprehension. Science 304, 438-441

6 Willems, R.M. et al. (2007) When language meets action: the neural integration of gesture and speech. Cereb. Cortex 17, 2322-2333

7 Hagoort, P. et al. Semantic unification. In The Cognitive Neurosciences IV (Gazzaniga, M.S., ed.), MIT press (in press)

8 Hagoort, P. (2005) On Broca, brain, and binding: a new framework. Trends Cogn. Sci. 9, 416-423

9 Mesulam, M.M. (1990) Large-scale neurocognitive networks and distributed processing for attention, language, and memory. Ann. Neurol. 28, 597-613

10 Willems, R.M. and Hagoort, P. (2007) Neural evidence for the interplay between language, gesture, and action: a review. Brain Lang. 101, 278 289

1364-6613/\$ - see front matter (๑) 2008 Elsevier Ltd. All rights reserved. doi:10.1016/j.tics.2008.12.001 Available online 14 February 2009 